\title{
Dielectric Properties of Polypeptides in Solution. II. Aggregation of Poly( $\gamma$-benzyl L-glutamate) in Various Helicogenic Solvents
}

\author{
Hiroshi Kihara \\ Department of Physics, Jichi Medical School, \\ 3311-1, Yakushiji, Minamikawachi, Tochigi, 329-04 Japan.
}

(Received October 26, 1976)

\begin{abstract}
The aggregation of poly( $\gamma$-benzyl L-glutamate) in dioxane, benzene, tetrahydrofuran, and cyclohexanone was studied by dielectric dispersion. The agregates of the side-by-side type were found to form in dioxane and benzene, but probably not in cyclohexanone and tetrahydrofuran. In contrast, the aggregates of the head-to-tail type were observed in tetrahydrofuran and cyclohexanone. The origin from which the molecular aggregates should appear is discussed with special consideration of the electrostatic interaction between the solute molecules.

KEY WORDS Dielectric Dispersion / Poly( $\gamma$-benzyl L-glutamate) /

Aggregation /
\end{abstract}

It has been reported that poly $(\gamma$-benzyl Lglutamate) (PBLG) forms aggregates in such helicogenic solvents as dioxane, ${ }^{1-5,13,20}$ benzene,${ }^{2,3,5}$ chlorobenzene, ${ }^{14}$ chloroform,${ }^{2,5,13}$ ethylene dichloride ${ }^{3 a, 5-8,13}$ (EDC), cyclohexanone ${ }^{17}$ $(\mathrm{CHN})$, and tetrahydrofuran ${ }^{17}$ (THF). Two aggregate types were proposed previously, one, a type of side-by-side aggregate with molecules arranged in an antiparallel fashion, and the other, a type of head-to-tail aggregate with molecules arranged in a parallel fashion. The former is referred to the "SS type" and the latter, the "HT type."

The purpose of this paper is first to investigate by dielectric dispersion what types of aggregates appear in typical helicogenic solvents.

In dioxane, both types of aggregates have been reported to be formed. ${ }^{1-4}$ Wada $^{1}$ suggested from dielectric dispersion data that both types coexist in dioxane. Watanabe ${ }^{2}$ and Powers and Peticolas $^{3}$ have used electric birefringence to show that at least the aggregate of SS type appears. On the contrary, Gupta, et al. ${ }^{4}$ maintain that only the HT type is formed. The discrepancy among these investigators remains to be solved.

In benzene, Watanabe ${ }^{2}$ and Powers and Peticolas $^{3}$ suggested that the aggregates of SS type were formed. In EDC, an HT type is formed, according to Powers and Peticolas, ${ }^{3 \mathrm{a}}$ Watanabe $^{7}$ and Kihara. ${ }^{6}$ Table I summarizes the results from previous investigations. The relationship between the aggregate type and solvent will be discussed more in detail in this paper.

The second purpose of this report is to clarify the reason of the aggregate formation. Watanabe ${ }^{7}$ implied that the aggregates of the HT type were formed due to hydrogen-bonding between amino and carbonyl residues at each end of the $\alpha$-helix. In contrast, the aggregates of SS type may have some relation with the dielectric nature of the solvent, considering the fact that the dielectric constant of the solvent having the aggregates of SS type are comparatively small. In this paper, the electrostatic interaction between the solutes will be calculated on the assumption that the interaction energy is smaller than thermal energy and the solute molecules are regarded as point dipoles.

\section{EXPERIMENTAL}

Two PBLG samples were used in the experiment. One was purchased from Kyowa Hakko Co. (Tokyo; catalogue code, HA-300). It was 
originally prepared by using hexylamine as the initiator and EDC as the solvent. The other, code number 7003 , as prepared by using sodium methoxide as the initiator and dioxane as the solvent. The molecular weight were estimated from the intrinsic viscosity data in dichloroacetic acid (DCA) as $2.4 \times 10^{4}$ (HA-300) and $7.4 \times 10^{4}$ (7003), by using the intrinsic viscosity-molecular weight relation of Teramoto, et al. ${ }^{11}$ Dielectric dispersion was measured by using a transformer bridge, Model TR-10C (Ando Denki Co., Tokyo) in the frequency range from 30 to $3 \mathrm{MHz}$. Use was made of a platinum concentric cylinder cell with an air capacitance of $4.71 \mathrm{pF}$, which was placed in a Dewer vessel containing a transformer oil and the temperature was maintained constant within $0.01{ }^{\circ} \mathrm{C}$. The cell was calibrated with benzene. Solvents were distilled by the usual method. The dipole moment per peptide residue, $\mu_{0}$ for aggregated solutes was estimated from the quantity $\beta$ obtainable from dielectric dispersion data by the Buckingham-ApplequistMahr equation ${ }^{9,10}$ (BAM equation);

$$
\frac{4 \pi N_{\mathrm{A}} C \beta f g}{3 k T}=\frac{\left(2 \varepsilon_{0}+1\right)\left(\varepsilon_{0}-n^{2}\right)}{2 \varepsilon_{0}+n^{2}}-\frac{\left(2 \varepsilon_{\infty}+1\right)\left(\varepsilon_{\infty}-n^{2}\right)}{2 \varepsilon_{\infty}+n^{2}}
$$

where $\varepsilon_{0}$ and $\varepsilon_{\infty}$ are the dielectric constants of the solution at the zero- and the infinite-freqency limits, respectively, $N_{\mathrm{A}}=$ Avogadro's number, $C=$ solute concentration in $g / \mathrm{m} l, k=$ Boltzmann's constant, $T=$ absolute temperature and $n=$ refractive index of the solution. Theoretically the quantities $f$ and $g$ depend on the molecular dimension of the solute and the dielectric constant and the refractive index of the solvent, but from a practical standpoint, they can be taken as constants. The values $f=2 / 3$ and $g=1$ were adopted in the present analysis. ${ }^{12}$ When the solute is nonassociated, $\beta$ is given by

$$
\beta=m \mu_{0}^{2} / M_{0}
$$

where $M_{0}=$ molecular weight of the solute per peptide residue, $\mu_{0}=$ dipole moment per peptide residue, and $m=$ degree of polymerization obtained by dividing the molecular weight determined above by $M_{0}$. According to Perrin, ${ }^{18}$ the critical frequency $f_{\mathrm{c}}$ for a prolate ellipsoid of revolution is represented by

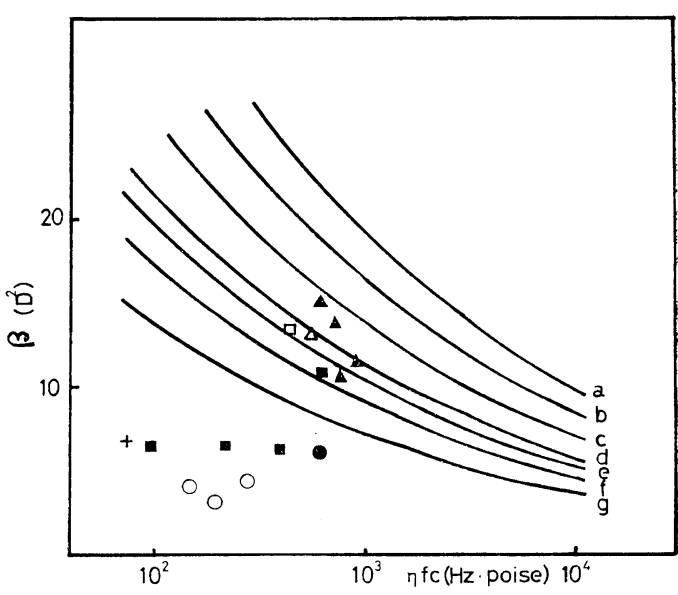

Figure 1. $\beta$ vs. $\eta f_{\mathrm{c}}$. The curves are calculated by eq 2 and $3, \mu_{0}$ as a parameter being. $\mu_{0}:(a)$, 6.5 D; (b), $6.0 \mathrm{D}$; (c), $5.5 \mathrm{D}$; (d), $5.0 \mathrm{D}$; (e), $4.8 \mathrm{D}$; (f), $4.5 \mathrm{D}$; (g), 4.0 D. The data shown in the figure were obtained in the mixed solvents: $\bigcirc$, dioxane-DMF; , dioxane- $m$-cresol; $\square$, benzeneDMF; $\square$, benzene- $m$-cresol; $\triangle$, EDC-DMF; $\boldsymbol{\Delta}$, EDC- $m$-cresol, sample code, HA-300, $25^{\circ} \mathrm{C}$; +, chloroform-DMF, sample code, 7003, $25^{\circ} \mathrm{C}$. Equation 1 was used to evaluate $\beta$ from the dielectric data.

$$
\begin{aligned}
f_{\mathrm{c}}= & \frac{3 k T \rho^{3}}{16 \pi^{2} a b^{2} \eta\left(1-\rho^{4}\right)} \\
& \times\left\{\frac{\left(2-\rho^{2}\right)}{\sqrt{1-\rho^{2}}} \log \frac{1+\sqrt{1-\rho^{2}}}{\rho}-1\right\}
\end{aligned}
$$

where

$$
\rho=a / b
$$

$2 a$ and $a b$ are the minor and the major axes of the ellipsoid, respectively, and $\eta$ is the viscosity of the solvent.

Values for $\eta f_{\mathrm{c}}$ where calculated by eq 3 with the reported result ${ }^{19}$ that $2 a=18.2 \AA$ and $2 b=$ $1.5 \AA \times$ (degree of polymerization). The solid curves in Figure 1 show the relationship between $\eta f_{\mathrm{c}}$ and $\beta$ calculated according to eq 2 an 3 , respectively, as a function of $\mu_{0}$. If an aggregate of the HT type is formed, the ordinate value will increase whereas $\eta f_{\mathrm{c}}$ will decrease, with the value of $\mu_{0}$ remaining constant. On the other hand, the formation of an aggregate of the SS type will lower both of these, and the value of $\mu_{0}$, estimated from eq 2 and $\beta$ obtained by eq 1 will appear smaller. Thus the type of 
aggregate formed may be determined from the location of the data point on the $\beta-\eta f_{\mathrm{c}}$ diagram. ${ }^{1}$

\section{RESULTS AND DISCUSSION}

Dielectric dispersion measurements were performed on PBLG dissolved in mixtures of two types of solvent; in one of these, (e.g., $m$-cresol or dimethylformamide (DMF)) PBLG is thought to be molecularly dispersed, whereas in the other (e.g., dioxane, benzene, CHN or THF) it is considered that PBLG forms aggregates. The former type of solvent is referred to as an aggegation-free solvent. The dipole moment per peptide residue, $\mu_{0}$, is calculated according to eq 1 and 2 from the experimental data for $\varepsilon_{0}$, $\varepsilon_{\infty}, C$, and $m$.

\section{The Aggregates in Dioxane}

The dipole moments per peptide residue, $\mu_{0}$, in mixtures of dioxane with DMF (or $m$-cresol) are plotted in Figure 2 as a function of the volume fraction of DMF (or $m$-cresol), where the values obtained in $m$-cresol and in an EDCDMF mixture are also plotted for the sake of reference. The latter solvents are typically aggregation-free. ${ }^{6,21}$ It is clearly shown that the dipole moments in dioxane and the dioxane mixtures are definitely smaller than those in the aggregation-free solvents. The critical frequencies obtained by the same experiments are plotted in Figure 1 as a function of $\beta$. According to the criterion described in the introduction, we can conclude that aggregates of SS type exist in dioxane and dioxane mixtures, in agreement with the conclusion of Wada ${ }^{1}$ Watanabe, ${ }^{2}$ and Powers and Peticolas. ${ }^{9}$ The conclusion of Gupta. et al., ${ }^{4}$ that the aggegate formed in the mixtures of dioxane and DCA would be of the HT type does not seem correct. However, the dipole moment per peptide residue in pure dioxane, which is calculated to be $2.5 \mathrm{D} /$ residue by the BAM equation from the data shown in Figure 1 of their article, is in good agreement with the present result, as shown in Figure 2. They also reported that the relationship between $\beta$ and $\eta f_{\mathrm{c}}$ in dioxane-DCA mixtures coincides well with that in chloroform-formamide mixtures in which the solutes are supposed to be in the molecularly dispersed state. As we have already reported, ${ }^{6}$ however, the dipole moments

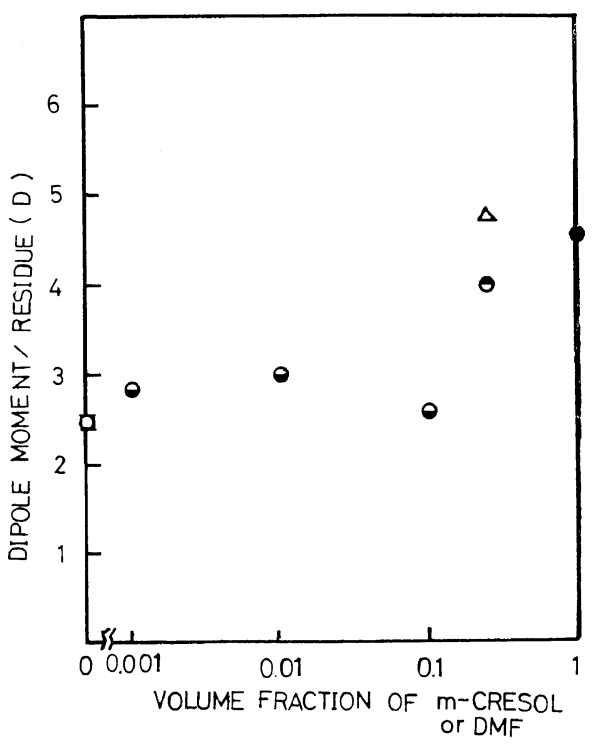

Figure 2. Dipole moment per peptide residue of PBLG in dioxane-DMF and dioxane- $m$-cresol mixtures as a function of the volume fraction of DMF and $m$-cresol: $\theta$, in dioxane-DMF, $25^{\circ} \mathrm{C}$; $\bigcirc$, in dioxane- $m$-cresol, $40^{\circ} \mathrm{C}$;, in pure $m$-cresol, $40^{\circ} \mathrm{C} ; \triangle$, in EDC- $m$-cresol- $(3: 1), 26^{\circ} \mathrm{C}$. In all the cases, the solute concentration was $0.36 \pm 0.09$ $(\mathrm{mg} / \mathrm{ml}) . \quad \square$, the value calculated by eq 1 , using the value of the dielectric increment shown in Figure 1 of the article by Gupta, et al. ${ }^{4}$

in chloroform-DMF mixtures were decidedly smaller than those in EDC-DMF mixtures indicating the degradation of the molecule by the presence of phosgene created from chloroform. Since the dipole moment per peptide residue obtained by Gupta, et al., in the chloroform-formamide mixtures is about the same as that obtained by the present author in chloroform-DMF, the mixtures of chloroform-formamide should be inadequate as aggegation-free solvents, and the dipole moment in dioxane must be compared with that in EDC-DMF or EDC$m$-cresol. Hence it is very probable that the dipole moment in dioxane and dioxane-DCA mixture is also smaller than that in the aggregation-free solvent.

Scharp ${ }^{15}$ found from dielectric dispersion measurements that the dipole moment per peptide residue was smaller in dioxane than in 1,2dichloroethene. He speculated that the smaller 
dipole moment in dioxane was due to the orientation of the side chain in antiparallel fashion. The dipole moment due to the carbonyl residue on the side chain of PBLG was reported by $\mathrm{Wada}^{16}$ to be antiparallel to the dipole moment of the main chain even in EDC, from the investigation of the copolymers of poly $(\gamma$-benzyl D-glutamate) and PBLG, and PBLG and PolyAla. It would then be more reasonable to consider that the apparent small dipole moment was due rather to the aggregates of the SS type.

\section{The Aggregates in Benzene}

The dipole moments per peptide residue in benzene-DMF (or $m$-cresol) mixtures are shown in Figure 3, which are also smaller than the value obtained in the aggregation-free solvents, but they increase with increasing volume fraction of DMF (or $m$-cresol). At least the aggregate of the SS type may exist in benzene- $m$-cresol mixtures, as is the case with dioxane. This conclusion is supported by the experimental data for $\beta$ and $\eta f_{\mathrm{c}}$ plotted in Figure 1. Watanabe ${ }^{2}$ and Powers and Peticolas ${ }^{9}$ already reported that the aggregates in the presence of benzene were

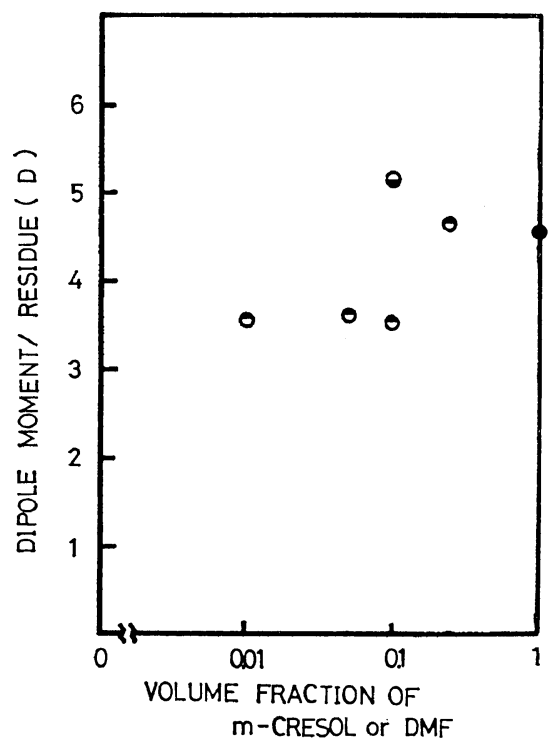

Figure 3. Dipole moment per peptide residue of PBLG in benzene-DMF and benzene- $m$-cresol mixtures as a function of the volume fraction of DMF and $m$-cresol: $\theta$, in benzene-DMF, $25^{\circ} \mathrm{C}$; $\ominus$, benzene- $m$-cresol, $34^{\circ} \mathrm{C}$;, in $m$-cresol, $40^{\circ} \mathrm{C}$. The solute concentration was $0.26 \pm 0.08(\mathrm{mg} / \mathrm{ml})$.

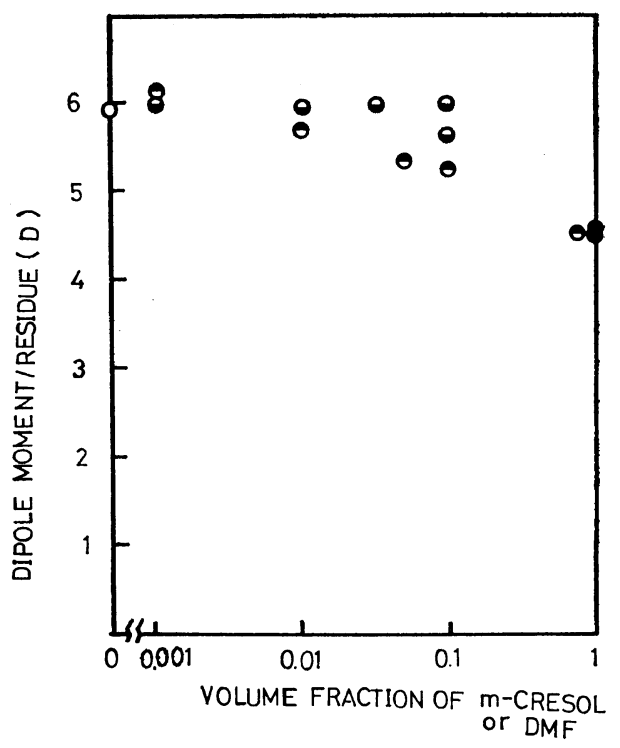

Figure 4. Dipole moment per peptide residue of PBLG in CHN-DMF and CHN- $m$-cresol mixtures as a function of the volume fraction of DMF and $m$-cresol: $\bigcirc$, in pure $\mathrm{CHN}, 25^{\circ} \mathrm{C}$; $\ominus$, in CHN-DMF, $25^{\circ} \mathrm{C}$; $\ominus$, in CHN-m-cresol, $25^{\circ} \mathrm{C}$; $O$, in $m$-cresol, $40^{\circ} \mathrm{C}$. The solute concentration was $0.37 \pm 0.03(\mathrm{mg} / \mathrm{ml})$.

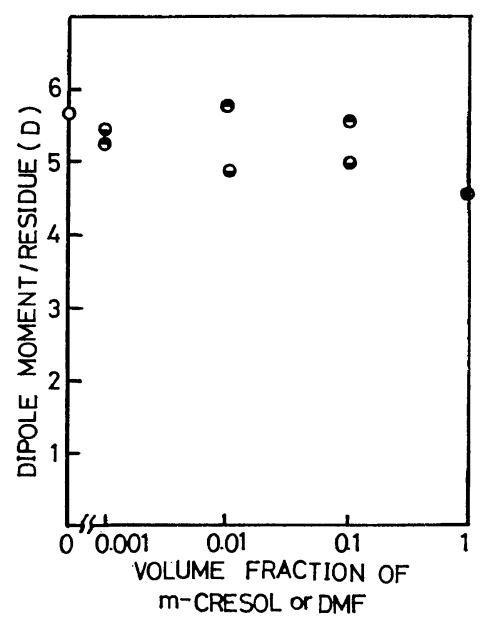

Figure 5. Dipole moment per peptide residue of PBLG in THF-DMF and THF $-m$-cresol mixtures as a function of the volume fraction of DMF and $m$-cresol: $\bigcirc$, in pure THF; $\ominus$, in THF-DMF; $\Theta$, in THF- $m$-cresol; $\Theta$, in pure $m$-cresol. The solute concentration was $0.38(\mathrm{mg} / \mathrm{ml}) ; 25^{\circ} \mathrm{C}$. 
of the SS type by the measurement of electric birefringence, in agreement with the present result.

Aggregates in Tetrahydrofuran and Cyclohexanone

The dipole moments per peptide residue obtained in THF-DMF (or $m$-cresol) mixtures are shown in Figure 4, which shows that the dipole moment per peptide residue is a little larger than the value obtained in the aggregation-free solvent and decreases with an increase in volume fraction. Similar results obtained for the mixed solvents of CHN and DMF (or $m$-cresol) are shown in Figure 5. The relationship between $\beta$ and $\eta f_{\mathrm{c}}$ obtained in THF-DMF (or $m$-cresol) and CHN-DMF (or $m$-cresol) mixtures are ploted in Figure 6. The results presented in Figures 4-6 indicate that in both the THF mixtures and the CHN mixtures the aggregates are thought to be of the HT type.

The Origin of the Side-by-Side Type Aggregates

The fact that the aggregates of the side-by-side

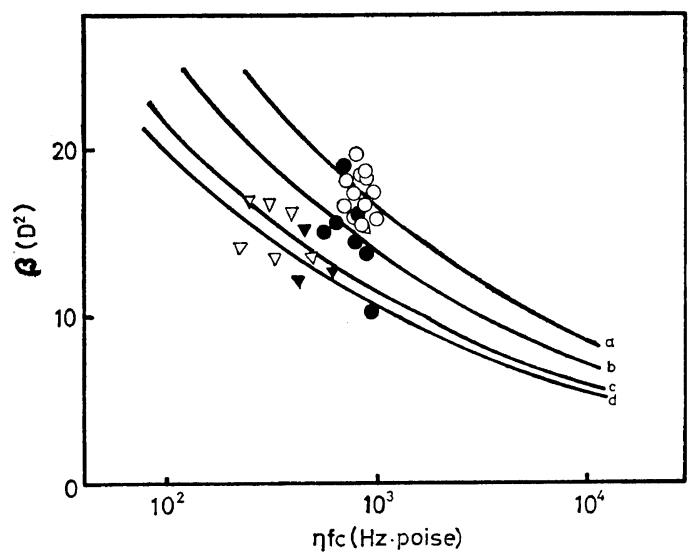

Figure 6. $\beta$ vs. $\eta f_{\mathrm{c}}$. The curves are calculated by eq 2 and 3. $\mu_{0}$ : (a), $6 \mathrm{D}$; (b), $5.5 \mathrm{D}$; (c), $5 \mathrm{D}$; (d), $4.8 \mathrm{D}$. Solvents: $\nabla, \mathrm{THF}-\mathrm{DMF} ; \boldsymbol{\mathrm { T }}, \mathrm{THF}-m-$ cresol; $\bigcirc, \mathrm{CHN}-\mathrm{DMF} ; \mathrm{O}, \mathrm{CHN}-m$-cresol. Sample used, $\mathrm{HA}-300 ; 25^{\circ} \mathrm{C}$. type exist only in such solvents with small dielectric constants as dioxane and benzene suggested that the electrostatic interaction between the solutes may play an important role in this type of the aggregation (see Table I). In order to confirm this, the order of magnitude of the dipole-dipole interaction in nonassociating solvents is estimated on the assumption that the interaction energy is less than thermal energy and the solutes are point dipoles.

Assuming that $N$ identical dipoles, each of whose dipole moment is $\mu$, exist in the volume $V$ under an applied electric field $E$, the average polarization per unit volume, $\langle\boldsymbol{P}\rangle$ can be written as

$$
\langle\boldsymbol{P}\rangle=\frac{1}{V} \int\left(\sum_{i=1}^{N} \mu_{i}\right) \exp \{-(w+u) / k T\} \frac{\prod_{i=1}^{N} \mathrm{~d} v_{i}}{(4 \pi V)^{N}}
$$

Here $\mu_{i}$ is the $i$-th dipole moment in the volume $V$, and

$$
\begin{gathered}
w=-\sum_{j=1}^{N}\left(\boldsymbol{\mu}_{j}^{*} \cdot \boldsymbol{E}\right) \\
u=\sum_{l=1}^{N} \sum_{k \neq l}^{N}\left\{\frac{\boldsymbol{\mu}_{l}}{\varepsilon} \cdot \frac{\partial}{\partial \boldsymbol{r}_{l k}} \frac{\left(\boldsymbol{\mu}_{k} \cdot \boldsymbol{r}_{l k}\right)}{r_{l k}^{3}}\right\}
\end{gathered}
$$

where $\boldsymbol{r}_{l k}$ represents the vector from the center of the $l$-th dipole to the center of the $k$-th dipole, $r_{l k}$ represents the absolute value of $\boldsymbol{r}_{l k}, \boldsymbol{\varepsilon}$ is the dielectric constant of the solvent, and $\mu_{j}{ }^{*}$ represents the effective $j$-th dipole moment, including the influence of the reaction field created by electric field $\boldsymbol{E}$.

Assuming both $w$ and $u$ are smaller than the thermal energy $k T$, eq 5 can be calculated as,

where

$$
\langle\boldsymbol{P}\rangle=\frac{n_{\mathrm{d}} \mu \mu^{*}}{3 k T} \boldsymbol{E}(1-\alpha)
$$

$$
\alpha=\frac{4 \pi n_{\mathrm{d}} \mu^{2}}{9 \varepsilon k T}
$$

Table I. The relationship between the dielectric constant and aggregate types

\begin{tabular}{lccc}
\hline \multicolumn{1}{c}{ Solvent } & Dielectric constant & Type of aggregates & References \\
\hline Dioxane & 2.27 & SS and HT & $1-4$ \\
Benzene & 2.28 & SS and HT(?) & 2,3 \\
Tetrahydrofuran & 7.1 & HT & This work \\
Ethylene dichloride & 10.2 & HT & 6,7 \\
Dichloroacetic acid & 18.2 & HT & This work \\
\hline
\end{tabular}


and $n_{\mathrm{d}}$ represents the number density. The details of the calculation appear in the appendix. When the dipole-dipole interaction is neglected, eq 8 becomes

$$
\langle\boldsymbol{P}\rangle=\frac{n_{\mathrm{d}} \mu \mu^{*}}{3 k T} \boldsymbol{E}
$$

which gives

$$
\Delta \varepsilon=\frac{4 \pi n_{\mathrm{d}} \mu \mu^{*}}{3 k T}
$$

where $\Delta \varepsilon=\varepsilon_{0}-\varepsilon_{\infty}$. When $\Delta \varepsilon \ll 1$, eq 1 can be approximately written

$$
\Delta \varepsilon=\frac{4 \pi N_{\mathrm{A}} b C m \mu_{0}^{2}}{9 k T M_{0}}
$$

where $b=2+n^{2} / \varepsilon_{0}{ }^{10} \quad$ By comparing eq $1^{\prime}, 8^{\prime}$, and $8, \Delta \varepsilon$, including the dipole-dipole interaction among the solutes, can be written

$$
\Delta \varepsilon=\frac{4 \pi N_{\mathrm{A}} b C m \mu_{0}^{2}}{9 k T M_{0}}(1-\alpha)
$$

where

$$
\alpha=\frac{4 \pi N_{A} C m \mu_{0}^{2}}{9 \varepsilon k T M_{0}}
$$

Assuming $m^{\prime}$ dipoles aggregate in a head-totail (linear) fashion, eq 10 and 11 may be replaced by

$$
\Delta \varepsilon=\frac{4 \pi N_{\mathrm{A}} \mathrm{Cbmm}^{\prime} \mu_{00}^{2}}{9 k T M_{0}}
$$

where $\mu_{00}$ represents the dipole moment per peptide residue in the molecularly dispersed state and

$$
\alpha^{\prime}=\frac{4 \pi N_{\mathrm{A}} C m m^{\prime}}{9_{\varepsilon} k T M_{0}} \mu_{0}^{2}
$$

The apparent dipole moment per peptide residue $\mu_{0}$ (app) obtained from eq $1^{\prime}$ without consideration of the aggregation, is then represented as

$$
\mu_{0}(\mathrm{app})=\mu_{00} \sqrt{m^{\prime}\left(1-\alpha^{\prime}\right)}
$$

Using the reported value of $4.8 \mathrm{D}$ for $\mu_{00},{ }^{8}$ values of $\mu_{0}(\mathrm{app})$ were calculated as a function of $C \varepsilon / T$. The solid curves in Figures 7 and 8 denote the values calculated for $m^{\prime}=1,2$, and 3 . If there exist the side-by-side type aggregates with the molecules arranged in an antiparallel fashion, the observed $\mu_{0}$ values would become smaller than those predicted by the solid curves in the figures. The experimentally obtained dipole moments per peptide residue (calculated according to eq 1) in dioxane-DMF, dioxane- $m$-cresol, benzene- $m$-cresol, and benzene-DMF are plotted $v s . C \varepsilon / T$ in Figure 7. It is seen that the experimental values of $\mu_{0}(\mathrm{app})$ seem smaller in the solvents with small dielectric constants than in the nonassociating solvents, and these values decrease with increasing $C \varepsilon / T$ for concentration range examined. From viscosity and light scattering measurements, Sakamoto, et al. ${ }^{17}$ have suggested that aggregates appear consisting of several molecules. The main attractive force forming such aggregates may be the Van der Waals force. The dipole-dipole interaction would further stabilize the aggregate of the SS type so formed.

Figure 8 shows the data for CHN-DMF, CHN- $m$-cresol, THF-DMF, and THF-mcresol solutions. The apparent dipole moments in these solvent mixtures are larger than those in the nonassociating solvents and do not vary appreciably with $C \varepsilon / T$.

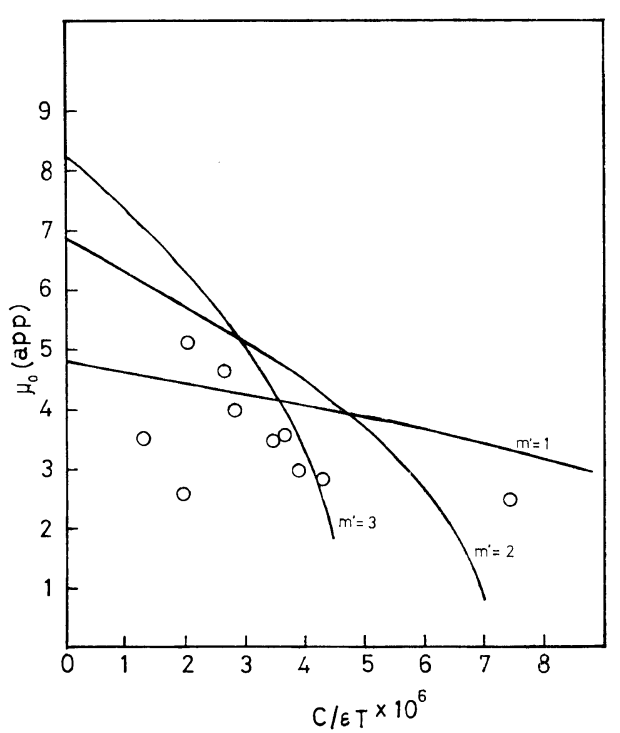

Figure 7. The observed dipole moment per peptide residue vs. $C / \varepsilon T$ in two types of solvent mixtures with varying solvent compositions; one is dioxane or benzene, and the other is DMF or $m$-cresol. The solid lines are derived by eq 11 . The parameter $m^{\prime}$ represents the number of the agregates in head-to-tail fashion (see text). The value of $4.8 \mathrm{D} /$ residue was adopted as $\mu_{00}$ (the dipole moment per peptide residue in the aggregation-free solvents $\left.^{6}\right)$. 


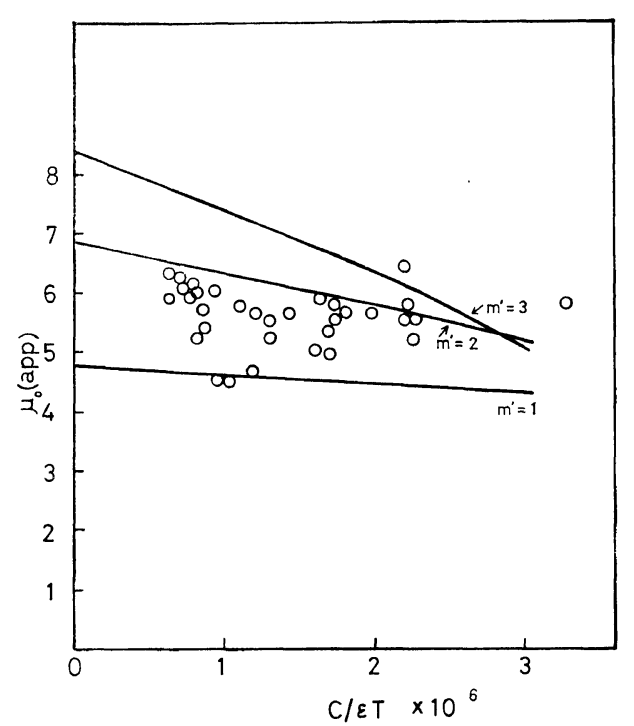

Figure 8. The observed dipole moment per peptide residue vs. $C / \varepsilon T$ in the mixtures of $C H N$ or THF with DMF or $m$-cresol. The solid lines are calculated by eq 11 . See the legend to Figure 7 for notation.

The Origin of the Head-to-Tail Type Aggregate

The aggregates of the HT type were reported to appear in dioxane, ${ }^{1,4}$ chloroform, ${ }^{2}$ EDC, ${ }^{3,6-8}$ and probably in benzene. Watanabe ${ }^{7}$ has proposed that these types of aggregates are formed due to the hydrogen bonding at each end of the solute molecules. The stability of the aggregates may depend on the relative strength of the hydrogen bond formed between the solvent and solute molecules and the hydrogen bond formed between the solute molecules, rather than on the dielectric constant of the solvent. In fact, the aggregates disappear in such solvents as DMF which can form hydrogen bonds with the chain ends of the solute molecules. This explanation is consistent with the fact that only the HT type aggregates were found in such solvents as CHN and THF, which have relatively large dielectric constants.

Acknowledgment. The author wishes to express his sincere appreciation to Professor O. Aono of Jichi Medical School for his fruitful comments and Professor A. Wada of the University of Tokyo for his continuous encouragement and useful comments throughout this work.
Thanks are due also to Miss K. Hamano for the measurements of the dielectric dispersion and the help in the data analysis.

This paper is dedicated to Professor Taro Kihara in honor of his sixtieth birthday.

\section{APPENDIX}

Since $u$ and $w$ are assumed to be small compared with $k T$, eq 5 can be expanded in a power series of $u / k T$ and $w / k T$. If we retain the first and second terms in the expansion, the polarization per volume $V$ may be written as

$$
\begin{aligned}
\langle\boldsymbol{P}\rangle= & \frac{1}{V}\left(\sum_{i=1}^{N} \boldsymbol{\mu}_{i}\right)\left\{1+\frac{\sum_{j=1}^{N}\left(\boldsymbol{\mu}_{j}^{*} \cdot \boldsymbol{E}\right)}{k T}\right\} \\
& \times\left[1-\frac{1}{k T} \sum_{l=1}^{N} \sum_{k \neq l}^{N}\left\{\boldsymbol{\mu}_{l} \cdot \frac{\partial}{\partial \boldsymbol{r}_{l k}}\left(\frac{\left(\boldsymbol{\mu}_{k} \cdot \boldsymbol{r}_{l k}\right)}{\varepsilon \boldsymbol{r}_{l k}^{3}}\right)\right\}\right] \\
& \times \frac{\prod_{i=1}^{N} \mathrm{~d} \Omega_{i} \prod_{i=1}^{N} \mathrm{~d} \boldsymbol{r}_{i}}{(4 \pi V)^{N}}
\end{aligned}
$$

which reduces to

$$
\begin{aligned}
\langle\boldsymbol{P}\rangle= & \frac{n_{\mathrm{d}} \mu \mu^{*}}{3 k T} E-\frac{n_{\mathrm{d}} N}{(k T)^{2}} \int \boldsymbol{\mu}_{1}\left(\boldsymbol{\mu}^{2 *} \cdot \boldsymbol{E}\right) \\
& \times\left(\boldsymbol{\mu}_{2} \cdot \frac{\partial}{\partial \boldsymbol{r}_{12}}\left(\frac{\mu_{1} \cdot \boldsymbol{r}_{12}}{\varepsilon r^{3}}\right)\right) \cdot \frac{\mathrm{d} \boldsymbol{r}_{1} \mathrm{~d} \boldsymbol{r}_{2} \mathrm{~d} \Omega_{1} \mathrm{~d} \Omega_{2}}{(4 \pi V)^{2}} \\
= & \frac{n_{\mathrm{d}} \mu \mu^{*}}{3 k T} \boldsymbol{E}-\frac{n_{\mathrm{d}}{ }^{2}}{(k T)^{2}} \int \boldsymbol{\mu}_{1}\left(\boldsymbol{\mu}_{2}{ }^{*} \cdot \boldsymbol{E}\right) \\
& \times\left(\boldsymbol{\mu}_{2} \cdot \frac{\partial}{\partial \boldsymbol{r}}\left(\frac{\left(\boldsymbol{\mu}_{1} \cdot \boldsymbol{r}\right)}{\varepsilon r^{3}}\right)\right) \cdot \frac{\mathrm{d} \boldsymbol{r} \mathrm{d} \Omega_{1} \mathrm{~d} \Omega_{2}}{(4 \pi)^{2}}
\end{aligned}
$$

In the last equation, $\boldsymbol{r}_{12}$ is simply given as $\boldsymbol{r}$.

It can be further simplified, by changing the volume integral to the surface integral, as

$$
\begin{aligned}
\langle\boldsymbol{P}\rangle= & \frac{n_{\mathrm{d}} \mu \mu^{*}}{3 k T} \boldsymbol{E}-\frac{{n_{\mathrm{d}}}^{2}}{(k T)^{2}} \int \frac{\mu_{1}\left(\mu_{2}{ }^{*} \cdot \boldsymbol{E}\right)}{\varepsilon} \frac{\mathrm{d} \Omega_{1} \mathrm{~d} \Omega_{2}}{(4 \pi)^{2}} \\
& \times \int\left(\mu_{2} \cdot \frac{r}{r}\right) \frac{\left(\mu_{1} \cdot \boldsymbol{r}\right)}{r^{3}} \mathrm{~d} S
\end{aligned}
$$

where $S$ means the surface area of the volume $V$. The integral over the small surface around the singular point at $r=0$ should vanish because of the finite excluded volume of the molecule.

The integral can then be performed to give

$$
\begin{aligned}
\langle\boldsymbol{P}\rangle= & \frac{n_{\mathrm{d}} \mu \mu^{*}}{3 k T} \boldsymbol{E}-\frac{n_{\mathrm{d}}{ }^{2}}{(k T)^{2}} \int \frac{\mathrm{d} \Omega_{1} \mathrm{~d} \Omega_{2}}{(4 \pi)^{2}} \cdot \frac{4 \pi \mu_{1}}{3 \varepsilon} \\
& \times\left(\boldsymbol{\mu}_{2}{ }^{*} \cdot \boldsymbol{E}\right)\left(\boldsymbol{\mu}_{1} \cdot \boldsymbol{\mu}_{2}\right)
\end{aligned}
$$




\section{H. KIHARA}

$$
=\frac{n_{\mathrm{d}} \mu \mu^{*} E}{3 k T}(1-\alpha)
$$

where $\alpha$ is given by eq 9 .

\section{REFERENCES}

1. A. Wada, J. Polym. Sci., 45, 145 (1960).

2. H. Watanabe, Nippon Kagaku Zasshi, (J. Chem. Soc. Jpn.), 86, 179 (1965).

3. (a) J.C. Powers and W. L. Poticolas, in "Ordered Fluids and Liquid Crystals" (Adv. in Chem. No. 63), J. Am. Chem. Soc., Washington D. C., 1967, p 217; (b) J. C. Powers, Jr. and W. L. Peticolas, Biopolymers, 9, 195, (1970).

4. A. K. Gupta, C. Dufour, and E. Marchal, ibid., 13, 1293 (1974).

5. P. Doty, J. H. Bradbury, and A. M. Holtzer, J. Am. Chem. Soc., 78, 947 (1956).

6. H. Kihara, Polym. J., 7, 406 (1975).

7. H. Watanabe, Nippon Kagaku Zasshi (J. Chem. Soc. Jpn.), 85, 403 (1964).

8. G. Boeckel, J. G. Genzling, G. Weill, and H. Benoit, J. Chim. Phys, 59999 (1962).
9. A. D. Buckingham, Australian J. Chem., 6 93, 323 (1953).

10. J. Applequist and T. G. Mahr, J. Am. Chem. Soc., 88, 5419 (1966).

11. A. Teramoto, K. Nakagawa, and H. Fujita, J. Chem. Phys., 46, 4197 (1967).

12. E. H. Ehrenrich and H. A. Scheraga, Macromolecules, 5, 746 (1972).

13. J. Gerber and H. G. Elias, Makromol. Chem., 112, 142 (1968).

14. V. J. Lilie, J. Springer, and K. Ueberreiter, Kolloid-Z. Z. polymere, 226, 138 (1968).

15. M. Scharp, J. Chem. Soc., Sect. A, 1596 (1970).

16. A. Wada, in "Poly $(\alpha$-amino acid $)$ s," G. Fasman Ed., Marcel Dekker, New York, N.Y., 1968, p 369.

17. (a) R. Sakamoto, N. Komori, T. Kojima, and M. Komori, The Report Read at the 23th Polymer Conference, Tokyo, Japan, 1974; (b) R. Sakamoto, et al., private communication.

18. F. Perrin, J. Phys. Radium, 5, 497, (1934).

19. A. Wada, J. Chem. Phys., 30, 329 (1959).

20. A. K. Gupta, Biopolymers, 15, 1543 (1976).

21. T. Matsumoto, N. Nishioka, A. Teramoto, and H. Fujita, Macromolecules, 7, 824 (1974). 